\title{
Perspiration Modelling of the Human Foot
}

\author{
Chris Breward ${ }^{1} \dagger$, and Andrew LaceY ${ }^{2}$ \\ ${ }^{1}$ University of Oxford, UK \\ ${ }^{2}$ Heriot-Watt University, UK \\ (Communicated to MIIR on 15 October 2021)
}

Study Group: ESGI 46, Mar 31-Apr 4, 2003, Bristol, UK

Communicated by: Chris Breward

Industrial Partner: SATRA Technology

Presenter: Tom Bayes

Team Members: Caroline Bird, Smith Institute; Chris Breward, University of Oxford; Jeff Dewynne, University of Oxford; Andrew Hill, University of Bath; Sam Howison, University of Oxford; Andrew Lacey, Heriot-Watt University; Colin Please, University of Southampton; John Ockendon, University of Oxford

Industrial Sector: Textile/Clothing/Footwear

Key Words: Footwear, Perspiration, Heat flow, Moisture flow

MSC2020 Codes: 80

\section{Summary}

SATRA Technology wishes to use an "artificial foot" to replace those of live volunteers in comfort tests for shoes. The artificial foot has the size and shape of a real foot and is made of a heated aluminium core surrounded by a polyurethane and a porous-rubber layer. The tests involve equipping the artificial foot with socks and the shoes or boots to be tested, and subjecting it to controlled environments for periods of up to several hours.

In this report, we build simple models for heat and moisture flow in an idealised foot. We consider two cases: "hot and sticky" (sweat layer present) and "cool and dry". We then build simple models for the wicking of liquid and temperature distribution in a sock. We find that wicking tends to lead to a uniform level of saturation across the sock.

$\dagger$ Corresponding Author: Chris.Breward@maths.ox.ac.uk 


\section{Summary}

The following report details two main themes:

1. Modelling of a bare foot. Two bare-foot models, for real and artificial feet, are discussed in $\S 2$. From a purely thermal point of view it transpires that the thermal properties of an artificial foot must be in reasonable agreement with those of a real foot if the experimental results for the two are to be similar under a variety of conditions. Inclusion of moisture leads to the further conclusion that to maintain the matching thermal properties, the porous rubber layer on the surface of the artificial foot should remain saturated.

2. Modelling of wicking and temperature within hose. A model for a sock, or other hose, is looked at in $\S 3$. This work must be regarded as preliminary as the sizes of a number of quantities and the boundary conditions (modelling heat and moisture transfer to the foot's environment) require further attention. Forced convection within the hose, resulting from foot movement, also has to be represented. It does appear that wicking tends to lead to a uniform level of saturation (dampness) across the hose. Some progress has been made in seeing how this saturation will evolve but the hose model needs to be coupled to another for outer footwear.

The problem of seeing how quantities scale, to be able to run faster experiments, has not been tackled.

\section{Introduction}

SATRA wish to use an "artificial foot", which has the size and shape of a real foot and is made of a heated aluminium core surrounded by a polyurethane and a porousrubber layer, to replace those of live volunteers in various "comfort" tests. These involve subjecting the "feet", wearing the hose (socks, stockings, ...) and shoes or boots being tested, to controlled environments for periods of up to several hours. It may be possible to get more repeatable experiments and to save time if an artificial foot can be used in testing how a foot's (surface) temperature varies and how moisture levels in the footwear can build up over time. For this method of footwear evaluation to work, the temperatures of the artificial foot's surface and of the corresponding genuine foot (assuming that the temperature of the outer skin is what is important) and the two "sweating" rates must agree reasonably well. A first step in checking this is to look at what happens to heat and moisture transfer for feet, real and artificial, exposed directly to the atmosphere. The next stage entails looking at mass and heat transport across the hose. Identification of key effects in a reasonable model might allow experiments to be carried out in different conditions from those really under investigation, and the desired temperatures and moisture levels determined from those measured. This might permit faster tests, say with greater temperature changes or moisture flows, to be used.

The investigations of the week concerned: 
- the modelling of how temperature and moisture vary in a SATRA artificial foot and in a real foot given various ambient conditions, to see how well an artificial foot might represent a real foot;

- the heat and mass transfer through and within a sock to try to establish key effects; similar modelling could apply to the porous-rubber layer of an artificial foot or to outer footwear.

In both parts, the layer of interest was regarded as a one-dimensional object although this might be somewhat unrealistic for the layers of an artificial foot, which has thickness of $\mathrm{O}(2 \mathrm{~cm})$ and other dimensions of $\mathrm{O}(10 \mathrm{~cm})$.

\section{The Bare Foot}

\subsection{Thermal problem only}

A real foot was taken to comprise a core part, in which a good supply of blood keeps the temperature maintained at the "body temperature" $T_{B}$, and a fairly thin "skin", which might also include a certain amount of sinew etc. The thickness of this layer is $\mathrm{O}(50 \mu \mathrm{m})$. Of course the body would act to regulate $T_{B}$ which might be reduced in cold conditions. Also, around the toes, for instance, the assumption of thinness is likely to be poor but the true qualitative behaviour is expected to be similar to what follows. With the skin regarded as thin, a one-dimensional model is appropriate. Also because of the small length scale and the time scale of interest, (hours), specific heat can be neglected to leave a simple linear equation for the surface temperature, $T_{S}$. This was regarded as the perceived temperature, that is, the temperature 'felt' by the foot. The temperature in the ambient air surrounding the foot is denoted by $T_{A}$. Initially, the effects of sweating are neglected and a purely thermal problem is considered.

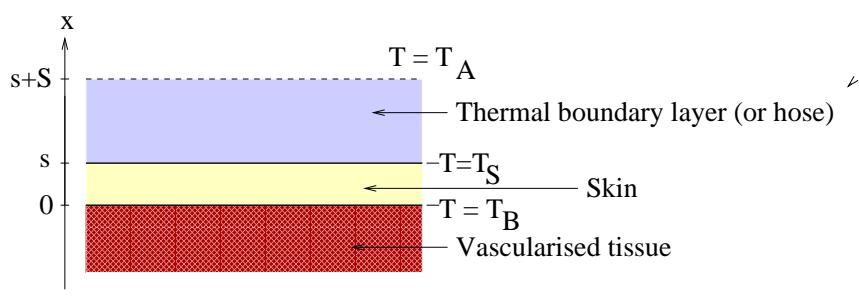

Figure 1: A simple one-dimensional picture for the outer part of a foot (allowing for possible footwear).

In Fig. 1 the region above the skin, i.e. $s<x<s+S$, represents some sort of thermal barrier between the skin of the foot and the air. This might be accounted for by a thermal boundary layer in air blowing past the foot but might also allow for, and be dominated by, the thermal resistance across hose and/or shoe. In any case, we assume an effective surface heat-transfer coefficient $h_{A}$ or, equivalently, a thermal resistance $R_{A}=1 / h_{A}$. Across the "skin", there is another thermal resistance $R_{B}=s / \kappa$, assuming a constant thermal conductivity $\kappa$ if $s$ is the skin thickness. (More generally, $R_{B}=\int(1 / \kappa) \mathrm{d} x$ if 
the thermal conductivity depends upon distance $x$ from the surface.) Equating the heat flows to and from the surface gives

$$
Q=\left(T_{B}-T_{S}\right) / R_{B}=\left(T_{S}-T_{A}\right) / R_{A}
$$

so

$$
T_{S}=\frac{R_{A} T_{B}+R_{B} T_{A}}{R_{A}+R_{B}}
$$

The artificial foot, with internal temperature $\hat{T}_{B}$ and internal thermal resistance $\hat{R}_{B}$, will have a corresponding surface temperature

$$
\hat{T}_{S}=\frac{R_{A} \hat{T}_{B}+\hat{R}_{B} T_{A}}{R_{A}+\hat{R}_{B}} .
$$

This means that to get a reasonable agreement between the (average) surface temperatures, for a variety of conditions, for instance allowing $T_{A}$ to change, the two internal (body) temperatures and resistances should be the same:

$$
\hat{T}_{B}=T_{B} \quad \text { and } \quad \hat{R}_{B}=R_{B} .
$$

Getting the latter to hold over the surface of a foot would mean that reasonable estimates of the thermal properties around the foot would have to be obtained. The former might be more difficult, since variation of $T_{B}$ with lateral position could not be copied as the aluminium core will have a spatially uniform temperature. Note that it is possible to mirror the body's response to a changing environment by altering the temperature of the aluminium core dynamically.

Of course this simple thermal analysis can be expected to give a very poor idea of the real situation as a quick order-of-magnitude calculation indicates that the total heat loss for a foot $(\mathrm{O}(5 \mathrm{~W}))$ is a similar size to the power needed to evaporate sweat (the rate of loss of moisture is $\mathrm{O}\left(6 \mathrm{gm} \mathrm{hr}^{-1}\right)$ so, taking a latent heat of $2.3 \times 10^{6} \mathrm{~J} \mathrm{~kg}^{-1}$, the associated power is $\mathrm{O}(4 \mathrm{~W}))$.

\section{$2.2 \quad$ A heat and moisture model}

In this section, the concept of cooling by sweating is introduced. Two cases might be envisaged: (A) "Hot and sticky", with a layer of sweat on the surface of the skin, see Fig. 2; (B) "Cool and dry" with any sweat produced from the pores being evaporated as it reaches the surface, see Fig. 3.

With $\rho_{w}=$ water density and perspiration rate $S(t)$, conservation of mass for the case (A) leads to the following equation for the thickness $h(t)$ of the sweat layer:

$$
\rho_{w} \frac{\mathrm{d} h}{\mathrm{~d} t}=-f\left(T_{S}\right)\left(p_{S}\left(T_{A}\right)-p_{v}(t)\right)+S(t)
$$

taking the evaporation rate to be a function of local temperature, that of the sweat layer, times the difference between the equilibrium partial pressure of water vapour, $p_{S}$, at that temperature and the (local) ambient partial pressure $p_{v}$. Note that it is assumed that the thickness $h$ is sufficiently small for the sweat to have the surface temperature $T_{S}$ and 


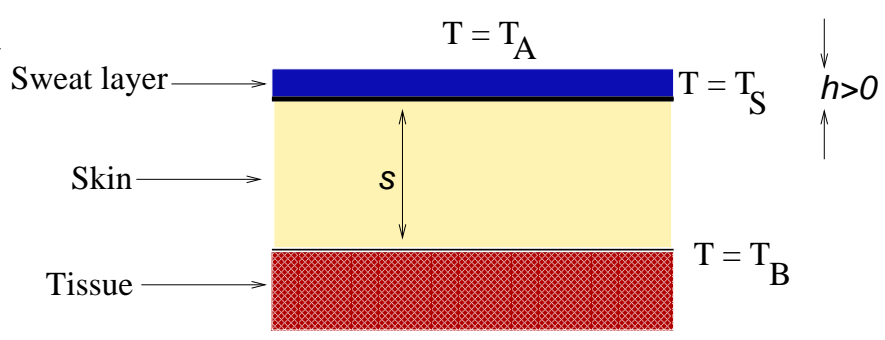

Figure 2: A layer of sweat of thickness $h$ lying over the skin of depth $s,(\mathrm{~A})$.

that it is this local temperaure which controls evaporation rate. As the heat problem is taken to be quasi-steady, so that the heat flux through the layer is constant, balancing heat flow in with that out (assuming Newtonian cooling) and that taken up through evaporation then leads to

$$
k_{s} \frac{\partial T}{\partial x}=k_{s}\left(T_{B}-T_{S}\right) / s=h_{A}\left(T_{S}-T_{A}\right)+L f\left(T_{S}\right)\left(p_{S}\left(T_{A}\right)-p_{v}(t)\right),
$$

where $k_{s}$ is the thermal conductivity, $h_{a}$ is the surface conductance of the air-liquid interface and $L$ is the latent heat of vaporisation. These hold as long as the layer of sweat exists, $h>0$, and also at an instant of $h=0$ if the right-hand side of (1) is positive.

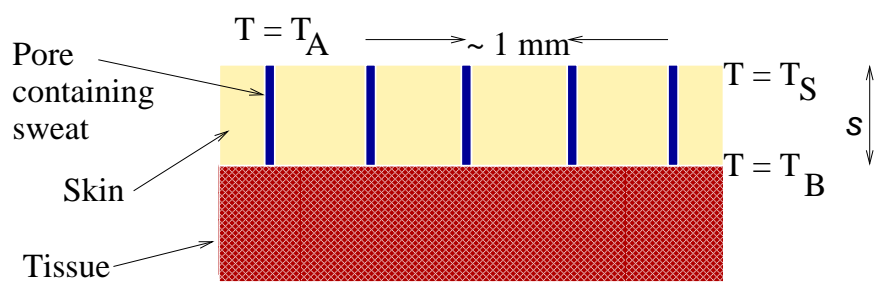

Figure 3: Sweat evaporating as it reaches the surface, (B).

If the sweat layer has vanished, $h=0$ at some time, it remains absent as long as the sweating rate is no greater than the maximum potential evaporation rate, $S(t) \leq f\left(T_{S}\right)\left(p_{S}\left(T_{A}\right)-p_{v}(t)\right)$. In this case the actual drying rate is simply $S(t)$ and then

$$
\begin{gathered}
k_{s} \frac{\partial T}{\partial x}=k_{s}\left(T_{B}-T_{S}\right) / s=H\left(T_{S}-T_{A}\right)+L S(t) \\
\text { so } \quad T_{S}=\frac{\left(k_{s} / s\right) T_{B}+H T_{A}-L S}{\left(k_{s} / s\right)+H} .
\end{gathered}
$$

The "skin" on the artificial foot is a layer, about $0.5 \mathrm{~cm}$ thick, of porous rubber. In place of sweat glands, water is fed into the base of the rubber through small tubes in the polyurethane which have a spacing of $4 \mathrm{~cm}$ or so (Fig. 4 (a)).

It is possible, in a dry environment, that the porous layer drys out and evaporation takes place well below the surface (Fig. 4 (a)). The depth of this evaporative surface could vary over the $4 \mathrm{~cm}$ scale. A suggestion was made that a semi-permeable membrane 
(a)

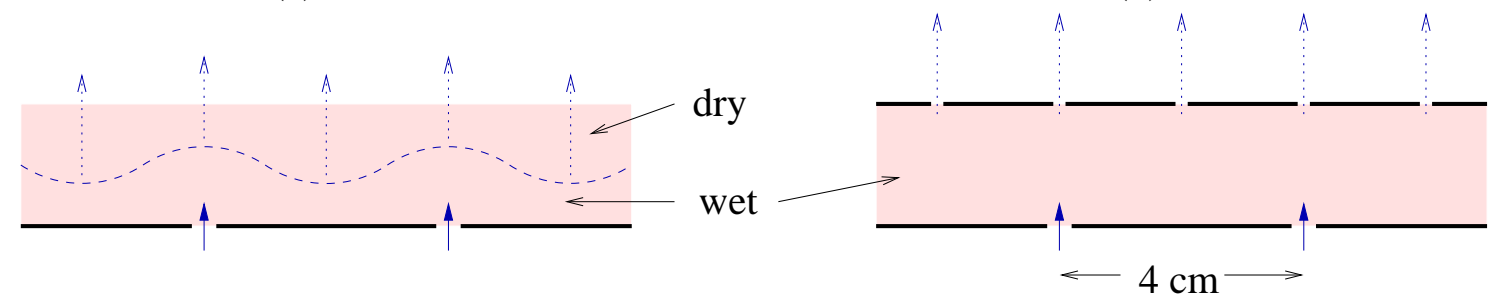

Figure 4: The porous rubber layer. (a) Evaporation might take place within the layer along some surface (the broken line), not necesarily flat. (b) An ideal case with some punctured sheet somehow preventing sub-surface evaporation and better mimicking a real skin.

put over the rubber could keep keep the porous rubber well saturated except near perforations; this would give a better representation of a real skin with pores. The view of SATRA was that this would probably not work. (A study along the lines of $\S 3$ might shed more light on this.) Of course, the likely spatially varying evaporation rate associated with a case like that shown in Fig. 4 (a), and the differing net heat transfer rates, resulting from differing wet and dry proportions, would lead to a heat flow which varies over the foot's surface. Perhaps of more significance is the possibility of the presence of a dry layer altering the average surface temperature because of a lower thermal conductivity (see (2)).

\section{Moisture and Heat Flow in Hose}

\subsection{The basic model}

We consider a model for a sock, or other hose, based on it being a thin porous medium. Similar models, but with vastly different parameter regimes, might hold for the porous rubber layer or for a shoe.

The following sizes were used in simplifying the model:

$(\mathcal{A} 1)$ Surface area of a foot $\approx$ area of hose $=\mathrm{O}\left(10^{-2} \mathrm{~m}^{2}\right)$;

$(\mathcal{A} 2)$ Thickness of hose $l \approx \mathrm{O}\left(10^{-3} \mathrm{~m}\right)$;

$(\mathcal{A} 3)$ Internal length scale for the hose $=\mathrm{O}\left(10^{-4} \mathrm{~m}\right)$;

$(\mathcal{A} 4)$ Variation of temperature, $T,=\mathrm{O}(10 \mathrm{~K})$;

$(\mathcal{A} 5)$ Associated (saturated) water-vapour partial pressure $p_{v}=\mathrm{O}\left(p_{S}\right)=$ $\mathrm{O}\left(10^{3} \mathrm{~N} \mathrm{~m}^{-2}\right)$

$(\mathcal{A} 6)$ Associated (saturated) water-vapour partial density $\rho_{v}=\mathrm{O}\left(\rho_{S}\right)=$ $\mathrm{O}\left(10^{-2} \mathrm{~kg} \mathrm{~m}^{-3}\right)$

$(\mathcal{A} 7)$ Air density $=\rho_{a}=\mathrm{O}\left(1 \mathrm{~kg} \mathrm{~m}^{-3}\right)$; it follows that the water mass fraction in the vapour phase is $\beta=\mathrm{O}\left(10^{-2}\right)$;

$(\mathcal{A} 8)$ Liquid water density $=\rho_{w}=\mathrm{O}\left(10^{3} \mathrm{~kg} \mathrm{~m}^{-3}\right)$; 
(A9) Specific heats of water (liquid \& vapour, $\left.c_{l} \& c_{v}\right)=\mathrm{O}\left(3 \times 10^{3} \mathrm{~J} \mathrm{~K}^{-1} \mathrm{~kg}^{-1}\right)$;

(A10) Latent heat of vaporisation $=L \approx 2 \times 10^{6} \mathrm{~J} \mathrm{~kg}^{-1}$;

$(\mathcal{A} 11)$ Surface tension $\approx 7 \times 10^{-2} \mathrm{~N} \mathrm{~m}^{-1}$;

$(\mathcal{A} 12)$ Typical thermal conductivity, $\kappa$, (between those of air and of liquid water $)=\mathrm{O}\left(10^{-1} \mathrm{~W} \mathrm{~m}^{-1} \mathrm{~K}^{-1}\right)$

$(\mathcal{A} 13)$ Typical observed rate of loss of heat from a foot $=\mathrm{O}(5 \mathrm{~W})$, i.e. the overall heat flux is $Q=\mathrm{O}\left(5 \times 10^{2} \mathrm{~W} \mathrm{~m}^{-2}\right)$;

$(\mathcal{A} 14)$ Typical observed rate of loss of sweat from a foot $=\mathrm{O}\left(6 \mathrm{gm} \mathrm{hr}^{-1}\right)$, i.e. the mass flux, as vapour, $q_{v}$, or as liquid, $q_{l}$, is $\mathrm{O}\left(10^{-4} \mathrm{~kg} \mathrm{~m}^{-2} \mathrm{~s}^{-1}\right)$;

$(\mathcal{A} 15)$ Mass diffusivities in gases and vapours, say $D$, are $\mathrm{O}\left(10^{-5} \mathrm{~m}^{2} \mathrm{~s}^{-1}\right)$; associated with these are coefficients $K_{i j}$ which give mass fluxes in terms of gradients of partial pressures, instead of gradients of densities (see (3), see (4) et seq.) and which are therefore $\mathrm{O}\left(10^{-10} \mathrm{~kg} \mathrm{~m} \mathrm{~N}^{-1} \mathrm{~s}^{-1}\right)$;

$(\mathcal{A} 16)$ "Total" water density, $\rho_{v}$, (averaged over a sock) associated with a time of hours is comparable with $(\mathcal{A} 8)$ so the liquid and gas volume fractions are expected to be $\mathrm{O}(1)$;

$(\mathcal{A} 17)$ With an air viscosity of $\mathrm{O}\left(10^{-5} \mathrm{Ns} \mathrm{m}^{-2}\right),(\mathcal{A} 3)$ and $(\mathcal{A} 7)$ indicate a Darcy "constant", $k_{g}$, for vapour flow of $\mathrm{O}\left(10^{-3} \mathrm{~kg} \mathrm{~m} \mathrm{~N} \mathrm{~N}^{-1} \mathrm{~s}^{-1}\right)$ (the precise figure will vary according to liquid volume fraction);

$(\mathcal{A} 18)$ The corresponding figure, $k_{l}$, (again depending upon saturation) for liquid water is $\mathrm{O}\left(10^{-2} \mathrm{~kg} \mathrm{~m} \mathrm{~N}^{-1} \mathrm{~s}^{-1}\right)$.

Flows of air and water vapour. The mass flows (across areas including the porous matrix) are expected to be of the form

$$
\begin{gathered}
\boldsymbol{q}_{a}=-(1-\beta) k_{g} \nabla p_{g}-\left(K_{a a} \nabla p_{a}+K_{a v} \nabla p_{v}\right) \\
\boldsymbol{q}_{v}=-\beta k_{g} \nabla p_{g}-\left(K_{v a} \nabla p_{a}+K_{v v} \nabla p_{v}\right)
\end{gathered}
$$

where the first terms on the right-hand sides are given by mass fractions times the total Darcy flow and the other terms are diffusive. Here $\beta$ is the mass fraction of water vapour in the gas phase, $p_{g}$ is the total pressure of the gas phase and is the sum of $p_{a}$, the partial pressure of the air (excluding water), and $p_{v}$, the partial pressure of the water vapour, $k_{g}$ is the Darcy "constant" referred to in $(\mathcal{A} 17)$, and the $K_{i j}$ s are related to diffusivities.

The total gas mass flux, $\boldsymbol{q}_{a}+\boldsymbol{q}_{v}$, should equal $-k_{g} \nabla p_{g}$. This holds if $K_{a a}=-K_{v a} \equiv$ $K_{a}$ and $K_{v v}=-K_{a v} \equiv K_{v}$. Writing $p_{a}=p_{g}-p_{v}$,

$$
\begin{gathered}
\boldsymbol{q}_{a}=-\left((1-\beta) k_{g}+K_{a}\right) \nabla p_{g}+\left(K_{a}+K_{v}\right) \nabla p_{v} \\
\boldsymbol{q}_{v}=-\left(\beta k_{g}-K_{a}\right) \nabla p_{g}-\left(K_{a}+K_{v}\right) \nabla p_{v} .
\end{gathered}
$$

The last term can be identified with a diffusive term, $-D \nabla \rho_{v}$, if $D=$ diffusivity $=\left(K_{a}+K_{v}\right) \frac{\partial p_{v}}{\partial \rho_{v}}$. With $\frac{\partial p_{v}}{\partial \rho_{v}} \sim \mathrm{O}\left(\frac{p_{v}}{\rho_{v}}\right)=\mathrm{O}\left(10^{5} \mathrm{Nm} \mathrm{kg}^{-1}\right), K=K_{a}+K_{v} \sim$ 


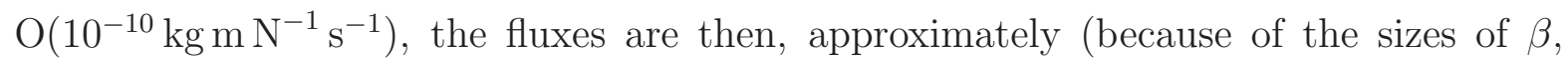
$k_{g}$ and the $\left.K_{i i} \mathrm{~s}\right)$,

$$
\boldsymbol{q}_{a}=-k_{g} \nabla p_{g}+K \nabla p_{v}, \quad \boldsymbol{q}_{v}=-\beta k_{g} \nabla p_{g}-K \nabla p_{v},
$$

where both $K$ and $k_{g}$ will depend upon the local saturation (liquid content).

Flow of liquid water. This mass flux takes the form

$$
\boldsymbol{q}_{l}=-k_{l} \nabla p_{l} \quad \text { with } p_{l}=p_{g}+\gamma
$$

where the term $\gamma$ is due to surface tension and controls the "wicking" of the material; from $(\mathcal{A} 11)$ and $(\mathcal{A} 3)$ it is expected to be $\mathrm{O}\left(7 \times 10^{-2} \mathrm{~N} \mathrm{~m}^{-1} / 10^{-4} \mathrm{~m}\right)=\mathrm{O}\left(10^{3} \mathrm{~N} \mathrm{~m}^{-2}\right)$. This term can depend upon saturation (as in the Buckley-Leverett equations) and temperature (giving a form of Marangoni convection).

Heat flow. The heat flux has conductive and convective parts:

$$
\boldsymbol{Q}=-\kappa \nabla T+\left(c_{l} \boldsymbol{q}_{l}+c_{v} \boldsymbol{q}_{v}+c_{a} \boldsymbol{q}_{a}\right) T .
$$

Here the $c_{i}$ s are specific heats.

Conservation and balance laws. It is convenient to take densities as averaged over total volumes so $\rho_{l}=$ liquid density $=\alpha \rho_{w}$ where $\alpha$ is the volume fraction occupied by liquid water and $\rho_{w}$ is usual water density, $\rho_{a}=$ "air density" $=(\varphi-\alpha) \rho_{I}$ where $\varphi$ is the porosity (i.e. $1-$ the solid volume fraction) and $\rho_{I}$ is the density of air within the gas phase, $\rho_{v}$ is the "vapour density" $=(\varphi-\alpha) \rho_{s}$ where $\rho_{s}$ is the density of vapour within the gas phase, and $\beta=\rho_{v} /\left(\rho_{a}+\rho_{v}\right)=\rho_{s} /\left(\rho_{I}+\rho_{s}\right)$. Writing the net rate of evaporation (mass/[time $\times$ volume (including pore and matrix)]) as $E$, laws for air, water vapour, liquid water and heat are

$$
\begin{gathered}
\frac{\partial \rho_{a}}{\partial t}+\nabla \cdot \boldsymbol{q}_{a}=0, \quad \frac{\partial \rho_{v}}{\partial t}+\nabla \cdot \boldsymbol{q}_{v}=E, \quad \frac{\partial \rho_{l}}{\partial t}+\nabla \cdot \boldsymbol{q}_{l}=-E \\
\text { and } \quad \frac{\partial}{\partial t}\left(\left(\rho_{h} c_{h}+\rho_{l} c_{l}\right) T\right)+\nabla \cdot \boldsymbol{Q}=-L E .
\end{gathered}
$$

Here $\rho_{h}$ is the density of the (dry) hose, assumed constant.

The evaporation rate will vanish if $p_{v}$ is the saturated vapour pressure for the local temperature: $p_{v}=p_{S}(T)$ (assuming that $\rho_{l}>0$ and that $p_{S}$ is not influenced by the nature of the hose). In general, $\left(p_{v}-p_{S}\right) E<0$. If evaporation and condensation can be considered to be very fast the precise form of $E$ becomes unimportant and instead we take $p_{v}=p_{S}(T)$ and $\rho_{s}=\rho_{S}(T)$. (The use of this approximation can mean that extra care has to be taken with boundary conditions; see below.) 


\subsection{Simplifying the model}

The evaporation rate can be eliminated to leave

$$
\begin{gathered}
\frac{\partial \rho_{a}}{\partial t}+\nabla \cdot \boldsymbol{q}_{a}=0, \quad \frac{\partial}{\partial t}\left(\rho_{l}+\rho_{v}\right)+\nabla \cdot\left(\boldsymbol{q}_{l}+\boldsymbol{q}_{v}\right)=0 \\
\text { and } \quad \frac{\partial}{\partial t}\left(\left(\rho_{h} c_{h}+\rho_{l} c_{l}\right) T+L \rho_{v}\right)+\nabla \cdot\left(\boldsymbol{Q}+L \boldsymbol{q}_{v}\right)=0
\end{gathered}
$$

with

$$
\begin{gathered}
\boldsymbol{q}_{a}=-k_{g} \nabla p_{g}+K \nabla p_{S}, \quad \boldsymbol{q}_{v}=-\beta k_{g} \nabla p_{g}-K \nabla p_{S}, \quad \boldsymbol{q}_{l}=-k_{l} \nabla\left(p_{g}+\gamma\right) \\
\text { and } \quad \boldsymbol{Q}=-\kappa \nabla T+\left(c_{l} \boldsymbol{q}_{l}+c_{v} \boldsymbol{q}_{v}+c_{a} \boldsymbol{q}_{a}\right) T
\end{gathered}
$$

as seven equations for the three densities, $\rho_{a}, \rho_{v}, \rho_{l}$, temperature $T$, and four fluxes $\boldsymbol{q}_{a}$, $\boldsymbol{q}_{v}, \boldsymbol{q}_{l}$ and $\boldsymbol{Q}$.

In addition, there are a number of constitutive laws: $p_{S}=p_{S}(T)$ from the watervapour-saturation assumption; $\rho_{S}=\rho_{S}(T)$ from an equation of state; $p_{g}=p_{S}+p_{a}$; and $p_{a}=p_{a}\left(\rho_{I}, T\right)$ from an equation of state; also

$$
\begin{array}{ccc}
\rho_{a}=(\varphi-\alpha) \rho_{I} ; & \rho_{v}=(\varphi-\alpha) \rho_{S} ; & \rho_{l}=\alpha \rho_{w} \\
\beta=\rho_{S} /\left(\rho_{S}+\rho_{I}\right) ; & k_{g}=k_{g}(\alpha) ; & k_{l}=k_{l}(\alpha) ; \\
K=K(\alpha) ; & \kappa=\kappa(\alpha) ; & \gamma=\gamma(\alpha, T) .
\end{array}
$$

The quantities $c_{h}$, the specific heat of hose, $c_{w}$, that of liquid water, $c_{v}$, that of water vapour, $c_{a}$, that of air, $\rho_{h}$, the density of hose, $\rho_{w}$, that of water, and $L$, the latent heat of vaporisation of water, are all regarded as constant.

This system of equations can be simplified considerably using sizes of various quantities in the list $(\mathcal{A} 1)-(\mathcal{A} 18)$. Since $\rho_{l} \gg \rho_{v}$, the conservation-of-water equation reduces to

$$
\frac{\partial \rho_{l}}{\partial t}+\nabla \cdot\left(\boldsymbol{q}_{l}+\boldsymbol{q}_{v}\right)=0 .
$$

The gas pressure can be eliminated from the first two fluxes to get, to leading order in $\beta$,

$$
\boldsymbol{q}_{a}=\left(\boldsymbol{q}_{v}+K \nabla p_{S}\right) / \beta
$$

Then

$$
\rho_{w} \frac{\partial \alpha}{\partial t}+\nabla \cdot\left(\boldsymbol{q}_{l}+\boldsymbol{q}_{v}\right)=0 \quad \text { and } \frac{\partial \rho_{a}}{\partial t}+\nabla \cdot\left(\frac{\boldsymbol{q}_{v}+K \nabla p_{S}}{\beta}\right)=0 .
$$

Given that the mass fluxes are $\mathrm{O}\left(10^{-4} \mathrm{~kg} \mathrm{~m}^{-2} \mathrm{~s}^{-1}\right)$, that the length scale is $\mathrm{O}\left(10^{-3}\right.$ $\mathrm{m})$, and that $\rho_{w} \approx 10^{3} \mathrm{~kg} \mathrm{~m}^{-3}$, the time scale associated with the first of these, the water equation, is $\mathrm{O}\left(10^{4} \mathrm{~s}\right)$ (i.e. a few hours). The term $K \nabla p_{S}$ is also $\mathrm{O}\left(10^{-4} \mathrm{~kg} \mathrm{~m}^{-2} \mathrm{~s}^{-1}\right), \beta$ is $\mathrm{O}\left(10^{-2}\right)$ and $\rho_{a}$ is $\mathrm{O}\left(1 \mathrm{~kg} \mathrm{~m}^{-3}\right)$ so a time scale for the second equation, the air equation, is indicated to be only $\mathrm{O}\left(10^{-1} \mathrm{~s}\right)$. This suggests that there is a rapid transient over which gas motion occurs. We are more interested in the longer time scale, on which the air equation reduces to the quasi-steady problem

$$
\nabla \cdot\left(\boldsymbol{q}_{v}+K \nabla p_{S}\right)=0
$$


Because we have an effectively one-dimensional problem, $\boldsymbol{q}_{v}+K \nabla p_{S}=A \boldsymbol{i}$, with $\boldsymbol{i}$ the unit vector in the $x$ direction (across the hose), and then (5) leads to $\partial A / \partial x=0$. Additionally, the air flux, $A$, vanishes on the skin surface, $x=0$, so (5) reduces to

$$
\boldsymbol{q}_{v}=-K \nabla p_{S} .
$$

Looking at the terms in the vapour-flux equation, written as

$$
\nabla p_{g}=-\left(\boldsymbol{q}_{v}+K \nabla p_{S}\right) / \beta k_{g}
$$

we see that the gradient of the gas pressure is $\mathrm{O}\left(10 \mathrm{~N} \mathrm{~m}^{-3}\right)$ and that this pressure only varies, over the length scale of $\mathrm{O}\left(10^{-3} \mathrm{~m}\right)$, by $\mathrm{O}\left(10^{-2} \mathrm{~N} \mathrm{~m}^{-2}\right)$. This indicates that the $p_{g}$ term is unimportant for the liquid flow and we are left with

$$
\boldsymbol{q}_{l}=-k_{g} \nabla \gamma
$$

The water problem is now just

$$
\rho_{w} \frac{\partial \alpha}{\partial t}=\nabla \cdot\left(k_{g}(\alpha) \nabla \gamma(\alpha, T)+K(\alpha) \nabla p_{S}(T)\right) .
$$

This gives us another quasi-steady problem since the dominant (and easily so) term of (6) is the first on the right-hand side:

$$
\nabla \cdot\left(k_{g}(\alpha) \nabla \gamma(\alpha, T)\right)=0 .
$$

This can be thought of as representing exremely good wicking. In fact, for the flux to be $\mathrm{O}\left(10^{-4} \mathrm{~kg} \mathrm{~m}^{-2} \mathrm{~s}^{-1}\right)\left(=\mathrm{O}\left(K \nabla p_{S}\right)\right)$, we need $\nabla \gamma(\alpha, T)=\mathbf{0}$, to leading order.

When we turn back to the heat equation,

$$
\frac{\partial}{\partial t}\left(\left(\rho_{h} c_{h}+\rho_{l} c_{l}\right) T+L \rho_{v}\right)=\nabla \cdot\left(\kappa \nabla T-L \boldsymbol{q}_{v}-\left(c_{l} \boldsymbol{q}_{l}+c_{v} \boldsymbol{q}_{v}+c_{a} \boldsymbol{q}_{a}\right) T\right),
$$

we see that the dominant two terms are the first two on the right-hand side so we simply get

$$
\nabla \cdot(\kappa(\alpha) \nabla T)=-L \nabla \cdot\left(K(\alpha) \nabla p_{S}(T)\right) .
$$

The one-dimensional model is now

$$
\begin{gathered}
\frac{\partial}{\partial x} \gamma(\alpha, T)=0 \\
\frac{\partial}{\partial x}\left(\kappa(\alpha) \frac{\partial T}{\partial x}+L K(\alpha) \frac{\partial}{\partial x} p_{S}(T)\right),
\end{gathered}
$$

with appropriate boundary conditions. Here $x$ is the distance from, say, the skin, so that $x=l$ corresponds to the outer surface of the hose, perhaps exposed to the air. Note that (8) says that the total heat flux (conductive + transport by vapour carrying latent heat), $Q=-\kappa(\alpha) \partial T / \partial x-L K(\alpha) \partial p_{S}(T) / \partial x$, does not vary with $x$. The mass equation, (7), says that $\gamma(\alpha, T)=\Gamma(t)$ for some $\Gamma$ and that if Marangoni effects can be neglected, i.e. $\gamma=\gamma(\alpha)$, then $\alpha=\alpha(t)$. For simplicity we assume this from now on, so the good wicking leads to a uniform saturation $\alpha$ across the hose.

It remains to determine how the saturation and heat flux evolve. 


\subsection{The boundary data}

Fitting in with the bare-foot work, $\S 2$, we suppose that Robin-type conditions hold at both surfaces of the hose:

$$
R_{B} \times \text { heat flux }\left.\right|_{x=0}=T_{B}-\left.T\right|_{x=0}, \quad R_{A} \times \text { heat flux }\left.\right|_{x=l}=\left.T\right|_{x=l}-T_{A} .
$$

Assuming no build-up of sweat at the skin's surface we also have

$$
\left.\left(q_{l}+q_{v}\right)\right|_{x=0}=q_{0} \quad \text { (specified) }
$$

For a well-ventilated (no-shoe) case, a possible second mass condition could be

$$
\left.p_{v}\right|_{x=l}=p_{A}=\text { partial pressure of water vapour in the neighbouring air. }
$$

Two possible difficulties arise:

$(\mathcal{B} 1)$ The last condition could conflict with the temperature condition (say if $\left.R_{A}=0\right)$ as the model requires $p_{v}=p_{S}(T)$; this suggests the possible presence of a boundary layer whose thickness is related to the speed of evaporation and condensation;

$(\mathcal{B} 2)$ A possible wrong interpretation of heat flux.

Taking (B2) first, the heat flux accounts for latent heat but not specific heat so $Q=-\kappa \partial T / \partial x+L q_{v}$ (above). The first temperature condition is then

$$
\left.Q\right|_{x=0}=\text { heat flux from the skin }=\left(T_{B}-\left.T\right|_{x=0}\right) .
$$

The second condition requires more care. If $T_{S}$ is the temperature right at the surface of the sock, as opposed to the temperature just in from any boundary layer indicated by $(\mathcal{B} 1)$, which we now denote by $T_{-}$,

$$
Q_{-}=\left(T_{S}-T_{A}\right) / R_{A}+L\left(q_{v-}+q_{l-}\right)=\text { total heat flow from the hose. }
$$

Here $Q_{-}$is the heat flow $Q$ just in from the boundary layer and $q_{v-}+q_{l-}$ is the corresponding total mass flux of water. It is again assumed that there is no buildup of moisture on the surface. This condition balances net conductive heat flow towards the surface with the power needed to evaporate the remaining liquid water.

The implications of $(\mathcal{B} 1)$ for the water condition at $x=l$ for our reduced model are hardest to deal with. The approximate vapour and heat equations,

$$
\frac{\partial \rho_{v}}{\partial t}=\frac{\partial}{\partial t}\left(K \frac{\partial p_{v}}{\partial x}\right)+E \quad, \quad \frac{\partial}{\partial t}\left(\left(c_{h} \rho_{h}+c_{l} \rho_{l}\right) T\right)=\frac{\partial}{\partial x}\left(\kappa \frac{\partial T}{\partial x}\right)-L E,
$$

can be reduced to

$$
\frac{\partial}{\partial t}\left(K \frac{\partial p_{v}}{\partial x}\right)+E=0 \quad, \quad \frac{\partial}{\partial x}\left(\kappa \frac{\partial T}{\partial x}\right)-L E=0
$$

in any boundary layer. 
Then, on scales appropriate to such a boundary layer,

$$
\kappa \frac{\partial T}{\partial x}+L K \frac{\partial p_{v}}{\partial x}=\text { const. }=0
$$

to match with the outer solution (above), applying in the interior of the hose. Because of the very good wicking, $\alpha$ is independent of position in the boundary layer so $K$ and $\kappa$ can both be regarded as constant and

$$
p_{v}=p_{A}+\frac{\kappa}{L K}\left(T_{S}-T\right) .
$$

It follows that

$$
\kappa \frac{\partial^{2} T}{\partial x^{2}}=L E\left(p_{A}+\frac{\kappa}{L K}\left(T_{S}-T\right), T\right),
$$

with $T=T_{S}$ on $x=l$ and $T \rightarrow T_{-}$as $x$ exits the boundary layer. For the matching condition and the differential equation to be consistent, $T_{-}$must be the solution of

$$
E\left(p_{A}+\frac{\kappa}{L K}\left(T_{S}-T_{-}\right), T_{-}\right)=0 .
$$

(There will be one, and only one, solution to this equation if $E\left(p_{v}, T\right)$ is an increasing function of $T$ and a decreasing function of $p_{v}$ with some saturation pressure $p_{S}(T)$, $E\left(p_{S}, T\right)=0$, corresponding to any $T$.)

This boundary-layer model has evaporation within the pores. If there is to be no extra evaporation at the very surface of the hose (this assumption might be one of several dubious ones), the only water flow at $x=l$ is the vapour flow $q_{v}=q_{S}=$ total water flux into, say, the air:

$q_{S}=q_{v-}+q_{l-}=-\left.K \frac{\partial p_{v}}{\partial x}\right|_{x=l}=\left.\frac{\kappa}{L} \frac{\partial T}{\partial x}\right|_{x=l}= \pm \frac{\kappa}{L} \sqrt{\frac{2 L}{\kappa} \int_{T_{-}}^{T_{S}} E\left(p_{A}+\frac{\kappa}{L K}\left(T_{S}-T\right), T\right) \mathrm{d} T}$,

which indicates a mass flux much larger, because of the appearance of $E$, than actually occurs.

The boundary layer cannot exist and $T_{x=l}=T_{S}$ must satisfy $p_{S}\left(T_{S}\right)=p_{A}$ (i.e. $\left.E\left(p_{A}, T_{S}\right)=0\right)$, say $T_{S}=T_{E}\left(p_{A}\right)$.

\subsection{The final problem}

The temperature problem is now

$$
\kappa(\alpha) \frac{\partial T}{\partial x}+L K(\alpha) \frac{\partial}{\partial x} p_{S}(T)=-Q(t) \quad \text { in } 0<x<l,
$$

with, from the boundary conditions,

$$
Q=\left(T_{B}-\left.T\right|_{x=0}\right) / R_{B}=\left(\left.T\right|_{x=l}-T_{A}\right) / R_{A}+L q_{S}
$$

and

$$
\left.T\right|_{x=l}=T_{S}=T_{E}\left(p_{A}\right) .
$$


From these four equations, $\left.T\right|_{x=0}$ is given by

$$
\kappa(\alpha)\left(T_{E}-\left.T\right|_{x=0}\right)+L K(\alpha)\left(p_{A}-p_{S}\left(\left.T\right|_{x=0}\right)\right)=-l Q=-l\left(T_{B}-\left.T\right|_{x=0}\right) .
$$

Thus $Q,\left.T\right|_{x=0},\left.T\right|_{x=l}$ and $q_{s}$ can all, at least in principle, be found in terms of the remaining dependent variable $\alpha$.

Then

$$
q_{s}=\left(Q-\left(T_{E}-T_{A}\right) / R_{A}\right) / L=\frac{1}{L}\left(\frac{T_{B}-\left.T\right|_{x=0}}{R_{B}}-\frac{T_{E}-T_{A}}{R_{A}}\right) \equiv q_{S}(\alpha) .
$$

Of course $\left.T\right|_{x=0}$ will depend upon the saturation $\alpha$ through the variation of $K(\alpha)$ (decreasing) and $\kappa(\alpha)$ (increasing).

Three different cases are possible:

$(\mathcal{C} 1) q_{S}(0) \geq q_{0}$; this means that the hose must remain dry, $\alpha \equiv 0$, i.e.; $\rho_{l} \equiv 0$, with $p_{v} \leq p_{S}(T)$, all evaporation actually occurs on the surface of the skin, and a simpler problem models the heat flow and temperature variation;

$(\mathcal{C} 2) q_{S}(0)<q_{0}$ but $q_{S}(\alpha)$ reaches $q_{0}$ for some $\alpha=A \leq \varphi$; in this case the saturation increases at the rate $\mathrm{d} \alpha / \mathrm{d} t=\left(q_{0}-q_{S}(\alpha)\right) / l \rho_{w}$ and, assuming smooth dependence of $q_{S}$, the hose never fully saturates (of course if $A=\varphi$ the hose gets arbitrarily wet);

$(\mathcal{C} 3) q_{S}(\alpha)<q_{0}$ for $0 \leq \alpha \leq \varphi$; in this case $\alpha$ would reach $\varphi$, and the hose becomes totally sodden.

Should Marangoni effects be important, $\gamma=\gamma(\alpha, T)$, the saturation $\alpha$ would depend upon position $x$ as well as time $t$. This would make the analysis of the problem harder but could leave the general behaviour unaltered.

\section{Further Remarks}

The above work has neglected effects of forced convection resulting from occasional compression (and subsequent re-expansion) of the hose during walking or because of other foot movement. With the above sizes, and taking the hose to be compressed significantly but with the compression only driving gas motion, an order-of-magnitude calculation suggests that this effect is comparable with the earlier diffusion-driven transport if the associated time scale is $l \rho_{v} / q_{s}=10^{-3} \mathrm{~m} \times 10^{-2} \mathrm{~kg} \mathrm{~m}^{-3} / 10^{-4} \mathrm{~kg} \mathrm{~m}^{-2} \mathrm{~s}^{-1}=10^{-1} \mathrm{~s}$, which would be an extremely rapid foot movement. Such forced convection, on the time scale associated with diffusion-driven air motion, would invalidate much of $\S 3$.

No work has so far been done on trying to use this type of modelling (with or without foot motion) to see how temperature and water supply can be increased to speed up experiments. 